\title{
Applications of Pell Polynomials in Rings
}

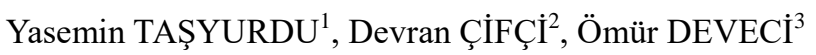 \\ ${ }^{1,2}$ Erzincan University, Department of Mathematics, Faculty of Science and Art, 24000 Erzincan, Turkey \\ ${ }^{3}$ Kafkas University, Department of Mathematics, Faculty of Science and Art, 36100 Kars, Turkey \\ Correspondence: Yasemin TAŞYURDU, Erzincan University, Department of Mathematics, Faculty of Science and Art, \\ 24000 Erzincan, Turkey.
}

Received: November 7, 2017

Accepted: : November 27, 2018 Online Published: March 26, 2018

doi:10.5539/jmr.v10n3p35

URL: https://doi.org/10.5539/jmr.v10n3p35

\begin{abstract}
In this paper, we study the Pell polynomials according to modulo $m$ where $x^{2}=2 x+1$ and various properties of these sequences are obtained. Also, Pell polynomials to the ring of complex numbers was defined. We define the Pell Polynomial-type orbits $P_{(\alpha, \beta)}^{R}(x)=\left\{x_{i}\right\}$ where $R$ be a 2-generator ring and $(\alpha, \beta)$ is a generating pair of the ring $R$. Furthermore, we obtain the periods of the Pell Polynomial-type orbits $P_{(\alpha, \beta)}^{R}(x)$ in finite 2-generator rings of order $p^{2}$.
\end{abstract}

2000 Mathematics Subjet Classification: 11B37, 11B83, 16 P10

Keywords: Pell polynomials, Period, Ring

\section{Introduction}

Integers sequences, such as Fibonacci, Lucas, Pell and Jacobsthal have been an intriguing topic for many years in Applied Mathematics. Many authors are dedicated to study this sequence, such as the work in (Deveci, 2015; Deveci \& Saraçoğlu Eskiyapar, 2016) and many other (Knox, 1992; K1lıç \& Taşç 2005). Fibonacci and Pell numbers are the most known numbers. Fibonacci sequence is defined by the equation $f_{n}=f_{n-1}+f_{n-2}$ where $f_{n}=f_{n-1}+f_{n-2}$ with the initial values $f_{0}=0, f_{1}=1, n \geq 2$ and $f_{n}$ are the terms of the sequence $0,1,1,2,3,5,8,13 \ldots$ The Pell sequence is defined recursively by the equation $P_{n+2}=2 P_{n-1}+P_{n}$ for $n \geq 0$ where $P_{0}=0$ and $P_{1}=1$. The Pell sequence is $0,1,2,5,12,29,70,169 \ldots$.

Most of the study of Fibonacci polynomials and Pell polynomials are applications in groups. D. D. Wall proposed the nation of Wall number of the Fibonacci sequence in 1960 and obtained many properties and theorems about these numbers (Wall 1960). Wilcox extend the problem to abelian groups (Wilcox 1986).

Fibonacci polynomials is defined as,

$$
f_{n}(x)=x f_{n-1}(x)+f_{n-2}(x) ; \quad n \geq 2 \text { with } f_{0}(x)=0, f_{1}(x)=1
$$

Lucas polynomials is defined as,

$$
l_{n}(x)=x l_{n-1}(x)+l_{n-2}(x) ; \quad n \geq 2 \text { with } l_{0}(x)=2, l_{1}(x)=1
$$

Pell polynomials is defined as,

$$
P_{n}(x)=2 x P_{n-1}(x)+P_{n-2}(x) ; \quad n \geq 2 \text { with } P_{0}(x)=0, P_{1}(x)=1
$$

Note the Pell polynomials are generated by matrix $P=\left(\begin{array}{cc}2 x & 1 \\ 1 & 0\end{array}\right)$,

$$
(P)^{n}=\left(\begin{array}{cc}
P_{n+1}(x) & P_{n}(x) \\
P_{n}(x) & P_{n-1}(x)
\end{array}\right)
$$

which can be proved by mathematical induction (Kılıç \& Taş̧ı 2005).

Pell-Lucas polynomials is defined as,

$$
Q_{n}(x)=2 x Q_{n-1}(x)+Q_{n-2}(x) ; \quad n \geq 2 \text { with } Q_{0}(x)=2, Q_{1}(x)=2 x
$$

D. J. DeCarli described the generalized Fibonacci sequences on an arbitrary ring in 1970 (DeCarli 1970). R. G. Buschman, A. F. Horadam and N. N. Vorobyov considered the set of integers for the special cases of these rings (Buschman, 1963; 
Horadam 1961; Vorobyov 1963). Also, O. Wyler studied with such a sequence on commutative ring with identity (Wyler 1965). Taşyurdu and Gültekin obtain the Fibonacci sequences of some rings of order $p^{2}$ by using recurrence $F_{n+2}=$ $A_{1} F_{n+1}+A_{0} F_{n}$ defined on the ring with identity, where $F_{0}=0$, the zero of the ring, $F_{1}=1$, the identity of the ring, $n \geq 0$ and $A_{0}, A_{1}$ are generator elements of the rings with identity and fields of order $p^{2}$. It was shown that these sequences are periodic and their periods are obtained (Taşyurdu \& Gültekin 2013; 2016). B. Fine gave the classification of all finite rings of order $p^{2}$ where $p$ a prime (Fine 1993). The period of generalized Fibonacci sequence in arbitrary rings has been obtained by Taşyurdu and Dilmen (Taşyurdu \& Dilmen, 2017). Also, they showed that determinant of Tridiagonal matrix derivated the terms of this sequence. Taşyurdu and Deveci study the Fibonacci polynomials in the ring of complex numbers and modulo $m$ (Taşyurdu \& Deveci 2017). If a sequence consists only of a fixed subsequence after a certain point, this sequence is called a periodic sequence. The period of this sequence is the number of terms in the repeating subsequence. The sequence is called a simple periodic sequence with $k$ period if the first $k$ terms in the sequence form a repeating subsequence (Knox, 1992).

For example, the sequence $\alpha, \beta, \mu, v, \gamma, \alpha, \beta, \mu, v, \gamma, \alpha, \beta, \mu, v, \gamma \ldots$ is simply periodic sequence. This sequence repeats with the initial element $\alpha$ and has period 5. The sequence $\alpha, \beta, \mu, v, \gamma, \beta, \mu, \nu, \gamma, \beta, \mu, v, \gamma \ldots$ is periodic with period 4 . The minimum period length of $\left(F_{i} \bmod n\right)_{i=-\infty}^{\infty}$ sequence is denote by $k(n)$ and is called Wall number of $n$ (Wall 1960).

\section{Consequence 1.1}

$$
\begin{gathered}
F_{k(n)} \equiv 0 \quad(\bmod n) \\
F_{k(n)-1} \equiv F_{k(n)+1} \equiv F_{k(n)+2} \equiv 1(\bmod n)
\end{gathered}
$$

(Renault, 1996).

From sequence (1.3) it can be obtained:

$$
\begin{gathered}
P_{k(n)} \equiv 0 \quad(\bmod n) \\
P_{k(n)-1} \equiv P_{k(n)+1} \equiv P_{k(n)+2} \equiv 1(\bmod n)
\end{gathered}
$$

Now we consider the following rings such that we will next address the periods of the Pell polynomials in these rings.

Theorem 1.2. For any prime $p$, up to isomorphism, the finite 2 -generator rings which is not field of order $p^{2}$ are given by the following presentations (Fine 1993).

$$
\begin{aligned}
& D=\left\langle\alpha, \beta \mid p \alpha=p \beta=0, \alpha^{2}=\alpha, \beta^{2}=\beta, \alpha \beta=\beta \alpha=0\right\rangle \cong \mathbb{Z}_{p}+\mathbb{Z}_{p} \\
& E=\left\langle\alpha, \beta \mid p \alpha=p \beta=0, \alpha^{2}=\alpha, \beta^{2}=\beta, \alpha \beta=\alpha, \beta \alpha=\beta\right\rangle \\
& F=\left\langle\alpha, \beta \mid p \alpha=p \beta=0, \alpha^{2}=\alpha, \beta^{2}=\beta, \alpha \beta=\beta, \beta \alpha=\alpha\right\rangle \\
& G=\left\langle\alpha, \beta \mid p \alpha=p \beta=0, \alpha^{2}=0, \beta^{2}=\beta, \alpha \beta=\alpha, \beta \alpha=\alpha\right\rangle \\
& H=\left\langle\alpha, \beta \mid p \alpha=p \beta=0, \alpha^{2}=0, \beta^{2}=\beta, \alpha \beta=\beta \alpha=0\right\rangle \cong \mathbb{Z}_{p}+C_{p}(0) \\
& I=\left\langle\alpha, \beta \mid p \alpha=p \beta=0, \alpha^{2}=\beta, \alpha \beta=0\right\rangle \\
& J=\left\langle\alpha, \beta \mid p \alpha=p \beta=0, \alpha^{2}=\beta^{2}=0\right\rangle \cong C_{p} \times C_{p}(0) .
\end{aligned}
$$

\section{Main Result}

\subsection{The Pell Polynomials Modulo $m$}

If we reduce the sequence of the Pell Polynomials by modulus $m$ such that $x^{2}=2 x+1$, we have a repeating sequence. This sequence is denoted by

$$
\left\{P_{n}(x)(\bmod m)\right\}=\left\{P_{0}(x)(\bmod m), P_{1}(x)(\bmod m), \ldots P_{i}(x)(\bmod m), \ldots\right\} .
$$

It has the same recurrence relation as in (1.3).

Theorem 2.1. The sequence $\left\{P_{n}(x)(\bmod m)\right\}$ is simply periodic.

Proof. Let $=\left\{\alpha_{1} x+\beta_{1}, \alpha_{2} x+\beta_{2} \mid 0 \leq \alpha_{1}, \alpha_{2}, \beta_{1}, \beta_{2} \leq m-1\right\}$, then $|S|=m^{4}$. The sequence $\left\{P_{n}(x)(\bmod m)\right\}$ repeats since there are only a finite number $m^{4}$ of pairs of terms possible, and the recurrence of a pair results in recurrence of all following terms, which implies that the sequence $\left\{P_{n}(x)(\bmod m)\right\}$ is periodic. Since the sequence is periodic, there exist natural numbers $i$ and $j$, with $i>j$ such that

$P_{i+1}(x)(\bmod m)=P_{j+1}(x)(\bmod m), P_{i+2}(x)(\bmod m)=P_{j+2}(x)(\bmod m)$. From the definition of the sequence $\left\{P_{n}(x)\right\}$, we can easily derive that $P_{n-2}(x)=P_{n}(x)-2 x P_{n-1}(x)$. Therefore, we obtain

$$
P_{i}(x)(\bmod m)=P_{j}(x)(\bmod m) \text {, }
$$

and hence, 


$$
\begin{gathered}
P_{i-1}(x)(\bmod m)=P_{j-1}(x)(\bmod m), \\
P_{i-2}(x)(\bmod m)=P_{j-2}(x)(\bmod m), \ldots \\
P_{i-j+1}(x)(\bmod m)=P_{1}(x)(\bmod m), \\
P_{i-j}(x)(\bmod m)=P_{0}(x)(\bmod m) .
\end{gathered}
$$

So we get that the sequence is simply periodic.

We next denote the period of the sequence $\left\{P_{n}(x)(\bmod m)\right\}$ by $h^{P(x)}(m)$.

Example 2.2. The sequence $\left\{P_{n}(x)(\bmod 3)\right\}$ is

$$
\{0,1,2 x, 2 x+2,2 x+1,0,2 x+1, x+1,2 x, 2,0,2, x, x+1, x+2,0, x+2,2 x+2, x, 1,0,1, \ldots\}
$$

and thus $h^{P(x)}(3)=20$.

For a given matrix $A=\left[P_{i j}\right]$ with $P_{i j}$ 's being polynomials, $A(\bmod m)$ means that every entry of $A$ is reduced modulo $m$, that is, $A(\bmod m)=\left(P_{i j}(\bmod m)\right)$. Let $\langle A\rangle_{m}=\left\{(A)^{n}(\bmod m) \mid n \geq 0\right\}$. If $\operatorname{gcd}(\operatorname{det} A, m)=1,\langle A\rangle_{m}$ is a cyclic group. We denote the cardinal of the set $\left\langle Q_{2}\right\rangle_{m}$ by $\left|\left\langle Q_{2}\right\rangle_{m}\right|$. Since $\operatorname{det} Q_{2}=-1$, it is clear that the set is a cyclic group for every positive integer $m$.

It is easy to see from (1.4) that $h^{P(x)}(p)=\left|\left\langle Q_{2}\right\rangle_{p}\right|$ for every prime $p$ if $x^{2}=2 x+1$.

Theorem 2.3. If $m=\prod_{i=1}^{t} p_{i}{ }^{e_{i}},(t \geq 1)$ where $p_{i}$ 's are distinct primes, then $h^{P(x)}(m)=\operatorname{lcm}\left[h^{P(x)}\left(p_{1}{ }^{e_{1}}\right), h^{P(x)}\left(p_{2}{ }^{e_{2}}\right), \ldots, h^{P(x)}\left(p_{t}{ }^{e_{t}}\right)\right]$.

Proof. Since $h^{P(x)}\left(p_{i}{ }^{e_{i}}\right)$ is the length of the period of the sequence $\left\{P_{n}(x)\left(\bmod p_{i}{ }^{e_{i}}\right)\right\}$, the sequence $\left\{P_{n}(x)\left(\bmod p_{i}{ }^{e_{i}}\right)\right\}$ repeats only after blocks of length $k \cdot h^{P(x)}\left(p_{i}{ }^{e_{i}}\right),(k \in \mathbb{N})$. Since also $h^{P(x)}(m)$ is the length of the period $\left\{P_{n}(x)(\bmod m)\right\}$, the sequence $\left\{P_{n}(x)\left(\bmod p_{i}{ }^{e_{i}}\right)\right\}$ repeats after $h^{P(x)}(m)$ terms for all values $i$. This implies that $h^{P(x)}(m)$ is of the form $k \cdot h^{P(x)}\left(p_{i}{ }^{e_{i}}\right)$ for all values of $i$. We thus prove that $h^{P(x)}(m)$ equals the least common multiple of $h^{P(x)}\left(p_{i}^{e_{i}}\right) l_{p}^{j}\left(u_{i}^{e_{i}}\right)$ 's.

Example 2.4. The sequences $\left\{P_{n}(x)(\bmod 2)\right\}$ and $\left\{P_{n}(x)(\bmod 6)\right\}$ are as follows, respectively:

$$
\{0,1,0,1, \ldots\}
$$

and

$$
\{0,1,2 x, 2 x+5,2 x+4,3,2 x+4,4 x+1,2 x, 5,0,5,4 x, 4 x+1,4 x+2,3,4 x+2,2 x+5,4 x, 1,0,1, \ldots\}
$$

Then, we obtain $h^{P(x)}(2)=2$ and $h^{P(x)}(6)=20$. Also we know that $h^{P(x)}(3)=20$. Thus it is verified that $h^{P(x)}(6)=\operatorname{lcm}\left[h^{P(x)}(2), h^{P(x)} 3\right]$.

Theorem 2.5. Let $p$ be a prime and let $u$ be the largest positive integer such that such that $h^{P(x)}(p)=h^{P(x)}\left(p^{u}\right)$. Then we have $h^{P(x)}\left(p^{v}\right)=p^{v-u} h^{P(x)}(p)$ for every $v \geq u$. In particular, if $h^{P(x)}(p) \neq h^{P(x)}\left(p^{2}\right)$, then we have $h^{P(x)}\left(p^{v}\right)=$ $p^{v-1} h^{P(x)}(p)$ for every $v \geq 2$.

Proof. Let $k$ be a positive integer and $I$ be the $2 \times 2$ identity matrix. If $\left(Q_{2}\right)^{h^{P(x)}\left(p^{k+1}\right)} \equiv I\left(\bmod p^{k+1}\right)$, then $\left(Q_{2}\right)^{h^{P(x)}\left(p^{k+1}\right)} \equiv I\left(\bmod p^{k}\right)$. This yields that $h^{P(x)}\left(p^{k}\right)$ divides $h^{P(x)}\left(p^{k+1}\right)$. Also, writing $\left(Q_{2}\right)^{h^{P(x)}\left(p^{k}\right)}=I+$ $\left(q_{i, j}^{(k)} \cdot p^{k}\right)$ we obtain

$$
\left(Q_{2}\right)^{h^{P(x)}\left(p^{k}\right) \cdot p}=\left(I+\left(q_{i, j}^{(k)} \cdot p^{k}\right)\right)^{p}=\sum_{i=0}^{p}\left(\begin{array}{c}
p \\
i
\end{array}\right)\left(q_{i, j}^{(k)} \cdot p^{k}\right)^{i} \equiv I\left(\bmod p^{k+1}\right)
$$

by the binomial expansion. This means that $h^{P(x)}\left(p^{k+1}\right)$ divides $h^{P(x)}\left(p^{k}\right) \cdot p$. Therefore, $h^{P(x)}\left(p^{k+1}\right)=h^{P(x)}\left(p^{k}\right)$ or $h^{P(x)}\left(p^{k+1}\right)=h^{P(x)}\left(p^{k}\right) \cdot p$, and the latter holds if, and only if, there is a $q_{i, j}^{(k)}$ which is not divisible by $p$. Since $h^{P(x)}\left(p^{u}\right) \neq h^{P(x)}\left(p^{u+1}\right)$, there is an $q_{i, j}^{(u+1)}$ which is not divisible by $p$, thus, $h^{P(x)}\left(p^{u+1}\right) \neq h^{P(x)}\left(p^{u+2}\right)$. To complete the proof we use an inductive method on $u$.

Example 2.6. Since $\left\{P_{n}(x)(\bmod 4)\right\}=\{0,1,2 x, 1,0,1, \ldots\}, h^{P(x)}(4)=4$. Also we know that $h^{P(x)}(2)=2$. Thus it is verified that $h^{P(x)}(4)=2 . h^{P(x)}(2)$.

\subsection{The Pell Polynomials in The Set of Complexs Numbers $\mathbb{C}$}

Define the sequence of the Pell polynomials in the set of complex numbers $\mathbb{C}$ as shown: 


$$
P_{n}=\left\{\begin{array}{lll}
0, & \text { if } & n=0 \\
1, & \text { if } & n=1 \\
2(i+1) P_{n-1}+P_{n-2} & \text { if } & n \geq 2
\end{array}\right.
$$

Letting

$$
M=\left[\begin{array}{cc}
2(i+1) & 1 \\
1 & 0
\end{array}\right]
$$

For $n \geq 1$, by (1.4) we may write

$$
M^{n}=\left[\begin{array}{cc}
P_{n+1} & P_{n} \\
P_{n} & P_{n-1}
\end{array}\right]
$$

It is easy to see that we obtain the sequence in (2.1) if we choose $x=2(i+1)$ in (1.3). We can get a repating sequence, by recuding the seguence of the Pell Polynomials in the set of complexs numbers $\mathbb{C}$ modulo $m$. It is denoted by

$$
\left\{P_{n}(x)(\bmod m)\right\}=\left\{P_{0}(x)(\bmod m), P_{1}(x)(\bmod m), \ldots P_{i}(x)(\bmod m), \ldots\right\}
$$

It has the same recurrence relation as in (1.4). Where $P_{i}^{m}(x)=P_{n}(x)(\bmod m)$. Let $h^{\mathbb{C}}(m)$ denote the period of the sequence of $P_{n}^{m}(x)$ in the set of complex numbers $\mathbb{C}$ modulo $m$ and $\left|\langle M\rangle_{m}\right|$ denote the order of the cyclic group $\langle M\rangle_{m}$ which is generated by reducing the matrix $m$ modulo. Therefore, it is clear that the rules produced for the period $h^{\mathbb{C}}(m)$ and the cyclic group $\langle M\rangle_{m}$ are of the same form of the results obtained in the above.

\subsection{The Pell Polynomials in Some Finite Rings}

Definition 2.3.1. We define the Pell Polynomial-type orbit $P_{(\alpha, \beta)}^{R}(x)=\left\{x_{i}\right\}$ of $(\alpha, \beta)$ by

$$
x_{0}=\alpha, \quad x_{1}=\beta, \quad x_{n+1}=2 \beta x_{n}+x_{n-1} \quad n \geq 1
$$

where $R$ be a 2 -generator ring and $(\alpha, \beta)$ be a generating pair of the ring $R$.

Similarly, we define the Pell Polynomial-type orbit $P_{(\beta, \alpha)}^{R}(x)=\left\{x_{i}\right\}$ of $(\beta, \alpha)$ by

$$
x_{0}=\beta, \quad x_{1}=\alpha, \quad x_{n+1}=2 \alpha x_{n}+x_{n-1} \quad n \geq 1 \text {. }
$$

Proposition 2.3.2. A Pell Polynomial-type orbit of a finite 2-generator is periodic.

Proof. Let $R$ be a finite 2-generator ring and $n$ be the order of $R$. Since there are $n^{2}$ distinct 2-tuples of elements of $R$, at least one of the 2-tuples appears twice in a Pell Polynomial-type orbit. Therefore, the subsequence following this 2-tuple repeats. Because of the repeating the sequence is periodic.

We next denote the period of the sequence $P_{(\alpha, \beta)}^{R}(x)$ by $P P_{(\alpha, \beta)}^{R}(x)$.

Definition 2.3.3. Let $R$ be a finite 2-generator ring. If there exist a Pell Polynomial-type orbit of the ring $R$ such that every element of the ring $R$ appears in the sequence, then the ring $\mathrm{R}$ is called Pell Polynomial-type sequenceable.

Proposition 2.3.4. For any prime $p \neq 2$,

$$
D=\left\langle\alpha, \beta \mid p \alpha=p \beta=0, \alpha^{2}=\alpha, \beta^{2}=\beta, \alpha \beta=\beta \alpha=0\right\rangle \cong \mathbb{Z}_{p}+\mathbb{Z}_{p}
$$

with generators $\alpha$ and $\beta$. The periods of the Pell Polynomial-type orbits $P_{(\alpha, \beta)}^{D}$ and $P_{(\beta, \alpha)}^{D}$ are $k(p)$.

Proof. Let us consider the Pell Polynomial-type orbit $P_{(\beta, \alpha)}^{D}$. The sequence $P_{(\beta, \alpha)}^{D}$ is as follows:

$$
\begin{gathered}
x_{0}=\beta, x_{1}=\alpha, x_{2}=2 \alpha+\beta, x_{3}=5 \alpha, x_{4}=12 \alpha+\beta, x_{5}=29 \alpha, \\
x_{6}=70 \alpha+\beta, x_{7}=169 \alpha, \ldots, x_{2 n}=P_{2 n} \alpha+\beta, x_{2 n+1}=P_{2 n+1} \alpha \\
x_{2 n+2}=2 \alpha x_{2 n+1}+x_{2 n}=2 \alpha\left(P_{2 n+1} \alpha\right)+\beta+P_{2 n} \beta=2 P_{2 n+1} \alpha^{2}+\beta+P_{2 n} \alpha \\
=2 P_{2 n+1} \alpha+\beta+P_{2 n} \alpha=\left(2 P_{2 n+1}+P_{2 n}\right) \alpha+\beta=P_{2 n+2} \alpha+\beta \\
x_{2 n+3}=2 \alpha x_{2 n+2}+x_{2 n+1}=2 \alpha\left(\beta+P_{2 n+2} \alpha\right)+P_{2 n+1} \alpha \\
=2 \alpha \beta+P_{2 n+2} \alpha^{2}+P_{2 n+1} \alpha=P_{2 n+2} \alpha+P_{2 n+1} \alpha=\left(P_{2 n+2}+P_{2 n+1}\right) \alpha \\
=P_{2 n+3} \alpha .
\end{gathered}
$$

Using the above, the sequence becomes:

$$
\beta+P_{0} \alpha, P_{1} \alpha, \beta+P_{2} \alpha, P_{3} \alpha, \ldots, \beta+P_{2 n} \alpha, P_{2 n+1} \alpha, \beta+P_{2 n+2} \alpha, P_{2 n+3} \alpha, \ldots,
$$

where $P_{n}$ denote the $n$ the term of the ordinary 2-step Pell sequence. Now let's determine the period of this sequence up to prime number $p$. It can be seen that the coefficient of the term $\beta$ of each element $x_{n}$ of this sequence is term of 
ordinary Pell sequences. Hence, the period of the sequence is determined by the coefficient of the term $\beta$ which $P_{n}$ is the $n$th term of the ordinary 2-step Pell sequence. Thus, we obtain $P P_{(\beta, \alpha)}^{D}(x)=k(p)$.

There is a similar proof for the sequence $P_{(\alpha, \beta)}^{D}(x)$.

Proposition 2.3.5. For any prime $p \neq 2$,

$$
F=\left\langle\alpha, \beta \mid p \alpha=p \beta=0, \alpha^{2}=\alpha, \beta^{2}=\beta, \alpha \beta=\beta, \beta \alpha=\alpha\right\rangle
$$

with generators $\alpha$ and $\beta$. The periods of the Pell Polynomial-type orbits $P_{(\alpha, \beta)}^{F}(x)$ and $P_{(\beta, \alpha)}^{F}(x)$ are $k(p)$.

Proof. Let us consider the Pell Polynomial-type orbit $P_{(\beta, \alpha)}^{F}(x)$. The sequence $P_{(\beta, \alpha)}^{F}(x)$ is as follows:

$$
\begin{gathered}
x_{0}=\beta, x_{1}=\alpha, x_{2}=2 \alpha+\beta, x_{3}=5 \alpha+2 \beta, x_{4}=12 \alpha+5 \beta, x_{5}=29 \alpha+12 \beta, x_{6}=70 \alpha+29 \beta, \\
x_{7}=169 \alpha+70 \beta, x_{8}=408 \alpha+169 \beta, \ldots, x_{n}=P_{n} \alpha+P_{n-1} \beta, x_{n+1}=P_{n+1} \alpha+P_{n} \beta, \\
x_{n+2}=2 \alpha x_{n+1}+x_{n}=2 \alpha\left(P_{n+1} \alpha+P_{n} \beta\right)+P_{n} \alpha+P_{n-1} \beta \\
=P_{n} 2 \alpha \beta+P_{n+1} 2 \alpha^{2}+P_{n-1} \beta+P_{n} \alpha=P_{n} 2 \beta+P_{n+1} 2 \alpha+P_{n-1} \beta+P_{n} \alpha \\
=\left(2 P_{n}+P_{n-1}\right) \beta+\left(2 P_{n+1}+P_{n}\right) \alpha=P_{n+1} \beta+P_{n+2} \alpha, \ldots \\
x_{n+3}=2 \alpha x_{n+2}+x_{n+1}=2 \alpha\left(P_{n+1} \beta+P_{n+2} \alpha\right)+P_{n} \beta+P_{n+1} \alpha \\
=P_{n+1} 2 \alpha \beta+P_{n+2} 2 \alpha^{2}+P_{n} \beta+P_{n+1} \alpha=P_{n+1} 2 \beta+P_{n+2} 2 \alpha+P_{n} \beta+P_{n+1} \alpha \\
=\left(2 P_{n+1}+P_{n}\right) \beta+\left(2 P_{n+2}+P_{n+1}\right) \alpha=P_{n+2} \beta+P_{n+3} \alpha=P_{n+3} \alpha+P_{n+2} \beta, \ldots
\end{gathered}
$$

Using the above, the sequence becomes:

$$
P_{0} \alpha+P_{-1} \beta, P_{1} \alpha+P_{0} \beta, P_{2} \alpha+P_{1} \beta, P_{3} \alpha+P_{2} \beta, \ldots, P_{n} \alpha+P_{n-1} \beta, P_{n+1} \alpha+P_{n} \beta, P_{n+2} \alpha+P_{n+1} \beta, \ldots
$$

where $P_{n}$ denote the $n$th term of the ordinary 2-step Pell sequence. That is, two consecutive terms of this sequences are $P_{n-1} \beta+P_{n} \alpha$ and $P_{n} \beta+P_{n+1} \alpha$. From Consequence 1.1., we have $P_{n-1} \beta+P_{n} \alpha \equiv \beta$ and $P_{n} \beta+P_{n+1} \alpha \equiv \alpha$ where $P_{n-1} \equiv 1$ and $P_{n+1} \equiv 1$. Thus, the sequence $P_{(\beta, \alpha)}^{F}(x)$ is periodic and $P P_{(\beta, \alpha)}^{F}(x)=k(p)$.

There is a similar proof for the sequence $P_{(\alpha, \beta)}^{F}(x)$.

Proposition 2.3.6. For any prime $p$, let

$$
G=\left\langle\alpha, \beta \mid p \alpha=p \beta=0, \alpha^{2}=0, \beta^{2}=\beta, \alpha \beta=\alpha, \beta \alpha=\alpha\right\rangle
$$

with generators $\alpha$ and $\beta$. The period of the Pell Polynomial-type orbit $P_{(\alpha, \beta)}^{G}(x)$ is $k(p)$ and $P_{(\beta, \alpha)}^{G}(x)$ is $2 p$.

Proof. It can clearly be seen that sequence created by Pell Polynomial-type orbit $P_{(\alpha, \beta)}^{G}(x)$ is similar to sequence created by Pell Polynomial-type orbit $P_{(\alpha, \beta)}^{F}(x)$. Thus, sequence Pell Polynomial-type orbit $P_{(\alpha, \beta)}^{G}(x)$ is periodic and $P P_{(\alpha, \beta)}^{G}(x)=k(p)$.

Let us consider the Pell Polynomial-type orbit $P_{(\beta, \alpha)}^{G}(x)$. The sequence $P_{(\beta, \alpha)}^{G}(x)$ is as follows:

$x_{0}=\beta, x_{1}=\alpha, x_{2}=\beta, x_{3}=3 \alpha, x_{4}=\beta, x_{5}=5 \alpha, x_{6}=\beta, x_{7}=7 \alpha, x_{8}=\beta, \ldots, x_{2 n}=\beta$,

$x_{2 n+1}=(2 n+1) \alpha$,

$x_{2 n+2}=2 \alpha x_{2 n+1}+x_{2 n}=\alpha((2 n+1) \alpha)+\beta=2(2 n+1) \alpha^{2}+\beta=\beta$

$x_{2 n+3}=2 \alpha x_{2 n+2}+x_{2 n+1}=2 \alpha \beta+(2 n+1) \alpha=2 \alpha+(2 n+1) \alpha=(2 n+3) \alpha, \ldots$

Using the above, the sequence becomes:

$$
\beta, \alpha, \beta, 3 \alpha, \beta, 5 \alpha, \beta, 7 \alpha, \beta, 9 \alpha, \ldots, \beta,(2 n+1) \alpha, \beta,(2 n+2) \alpha, \ldots
$$

Notice that each element $x_{n}$ of this sequence has the form

$$
x_{n}=\left\{\begin{array}{cl}
\beta & n=2 m \\
(2 m+1) \alpha & n=2 m+1
\end{array}\right.
$$

It can be seen that the period of the sequence is determined by prime number $p$. The residue class has $p$ elements according to modulo $p \geq 2$ and there are $\beta$ of term $p$ times. Thus, the sequence $P_{(\beta, \alpha)}^{G}(x)$ is periodic and $P P_{(\beta, \alpha)}^{G}(x)=2 p$.

Proposition 2.3.7. For any prime $p \neq 2$,

$$
H=\left\langle\alpha, \beta \mid p \alpha=p \beta=0, \alpha^{2}=0, \beta^{2}=\beta, \alpha \beta=\beta \alpha=0\right\rangle \cong \mathbb{Z}_{p}+C_{p}(0)
$$


with generators $\alpha$ and $\beta$.The period of the Pell Polynomial-type orbit $P_{(\alpha, \beta)}^{H}(x)$ is $k(p)$ and $P_{(\beta, \alpha)}^{H}(x)=2$.

Proof. It can clearly be seen that sequence created by Pell Polynomial-type orbit $P_{(\alpha, \beta)}^{H}(x)$ is similar to sequence created by Pell Polynomial-type orbit $P_{(\alpha, \beta)}^{D}(x)$. Thus, sequence of Pell Polynomial-type orbit $P_{(\alpha, \beta)}^{H}(x)$ is periodic and $P P_{(\alpha, \beta)}^{H}(x)=k(p)$.

Let us consider the Pell Polynomial-type orbit $P_{(\beta, \alpha)}^{H}(x)$. The sequence $P_{(\beta, \alpha)}^{H}(x)$ is as follows:

$x_{0}=\beta, x_{1}=\alpha, x_{2}=\beta, x_{3}=\alpha, x_{4}=\beta, x_{5}=\alpha, x_{6}=\beta, x_{7}=\alpha, x_{8}=\beta, \ldots, x_{2 n}=\beta, x_{2 n+1}=\alpha$,

$x_{2 n+2}=2 \alpha x_{2 n+1}+x_{2 n}=2 \alpha(\alpha)+\beta=\alpha^{2}+\beta=\beta$

$x_{2 n+3}=2 \alpha x_{2 n+2}+x_{2 n+1}=2 \alpha(\beta)+\alpha=\alpha$

Using the above, the sequence becomes:

$$
\beta, \alpha, \beta, \alpha, \beta, \alpha, \beta, \alpha, \beta, \alpha, \ldots, \alpha, \beta, \alpha, \ldots
$$

Notice that each element $x_{n}$ of this sequence has the form

$$
x_{n}= \begin{cases}\beta & n=2 m \\ \alpha & n=2 m+1\end{cases}
$$

The sequence in this ring consist only of repetitions of a fixed subsequence and the number of terms in the repeating subsequence is 2 . Thus, the sequence $P_{(\beta, \alpha)}^{H}(x)$ is periodic and $P P_{(\beta, \alpha)}^{H}(x)=2$.

Proposition 2.3.8. For any prime $p \neq 2$,

$$
I=\left\langle\alpha, \beta \mid p \alpha=p \beta=0, \alpha^{2}=\beta, \alpha \beta=0\right\rangle
$$

with generators $\alpha$ and $\beta$. The period of the Pell Polynomial-type orbit $P_{(\beta, \alpha)}^{I}(x)$ is $2 p$.

Proof. Let us consider the Pell Polynomial-type orbit $P_{(\beta, \alpha)}^{I}(x)$. The sequence $P_{(\beta, \alpha)}^{I}(x)$ is as follows:

$x_{0}=\beta, x_{1}=\alpha, x_{2}=3 \beta, x_{3}=\alpha, x_{4}=5 \beta, x_{5}=\alpha, x_{6}=7 \beta, x_{7}=\alpha, x_{8}=9 \beta, \ldots, x_{2 n}=(2 n+1) \beta, x_{2 n+1}=\alpha$,

$x_{2 n+2}=2 \alpha x_{2 n+1}+x_{2 n}=2 \alpha[\alpha]+(2 n+1) \beta=2 \beta+(2 n+1) \beta=(2 n+3) \beta$,

$x_{2 n+3}=2 \alpha x_{2 n+2}+x_{2 n+1}=2 \alpha(2 n+2) \beta+\alpha=2 \alpha \beta(2 n+2)+\alpha=\alpha$

Using the above, the sequence becomes:

$$
\beta, \alpha, 3 \beta, \alpha, 5 \beta, \alpha, 7 \beta, \alpha, 9 \beta, \alpha, \ldots,(2 n+1) \beta, \alpha \ldots
$$

Notice that each element $x_{n}$ of this sequence has the form

$$
x_{n}=\left\{\begin{array}{cl}
(2 n+1) m & n=2 m \\
\alpha & n=2 m+1
\end{array}\right.
$$

It can be seen that the period of the sequence is determined by prime number $p$. The residue class has $p$ elements according to modulo $p \geq 2$ and there are $\beta$ of term $p$ times. Thus, the sequence $P_{(\beta, \alpha)}^{I}(x)$ is periodic and $P P_{(\beta, \alpha)}^{I}(x)=2 p$.

\section{References}

Buschman, R. G. (1963). Fibonacci Numbers, Chebyshev Polynomials, Generalizations and Difference Equations. Fibonacci Quart., 1(4), 1-7.

DeCARLI, D. J. (1970). A Generalized Fibonacci Sequence Over An Arbitrary Ring. Fibonacci Quart. 8(2), 182-184, 198.

Deveci, Ö. (2015). The Pell-padovan sequences and The Jacobsthal-Padovan seuences in finite groups. Util. Math., 98 257-270.

Deveci, Ö. \& Saraçoğlu Eskiyapar, E. (2016). On The Pell Polynomials and The Pell Sequences in Groups. Chiang Mai J. Sci., 43, 247-256.

Fine, B. (1993). Classification of Finite Rings of Order $p^{2}$. Math. Mag., 66(4), 248-252. https://doi.org/10.2307/2690742

Horadam, A. F. (1961). A Generalized Fibonacci Sequence. Amer. Math. Monthly, 68(5), 445-459. https://doi.org/10.1080/00029890.1961.11989696

Kılıç, E. \& Taşçı, D. (2005). The Linear Algebra of the Pell Matrix. Bol. Soc. Mat. Mexicana, 3. 
Knox, S. W. (1992). Fibonacci Sequences in Finite Groups. Fibonacci Quart., 30(2), 116-120.

Renault, M. (1996). The Fibonacci Sequence Under Various Modulo. Master's Thesis. Wake Forest University.

Taşyurdu, Y. \& Gültekin, İ. (2013). On period of Fibonacci sequences in finite rings with identity of order $\mathrm{p}^{\wedge} 2$. Journal of Mathematics and System Science, 3, 349-352.

Taşyurdu, Y. \& Gültekin, İ. (2016). The period of Fibonacci sequences over the finite field of order $\mathrm{p}^{\wedge} 2$. New Trends in Mathematical Sciences, 4, 248-255. https://doi.org/10.20852/ntmsci.2016116033

Taşyurdu, Y. \& Dilmen, Z. (2017). On Period of Generalized Fibonacci Sequence Over Finite Ring and Tridiagonal Matrix. Celal Bayar University Journal of Science, 13(1), 165-169.

Taşyurdu, Y. \& Deveci, Ö. (2017). The Fibonacci Polynomials in Rings. Ars Combinatoria, 133, 355-366. https://doi.org/10.18466/cbayarfbe.302663

Vorobyov, N. N. (1963). The Fibonacci Numbers, translated from the Russian by Normal D. Whaland, Jr., and Olga A. Tittlebaum, D. C. Heath and Co., Boston.

Wall, D. D. (1960). Fibonacci series modulo m. Amer. Math. Monthly, 67(6), 525-532. https://doi.org/10.1080/00029890.1960.11989541

Wilcox, H. J. (1986). Fibonacci sequences of period n in groups. Fibonacci Quart. 24(4), 356-361.

Wyler, O. (1965). On Second-order Recurrences. Amer. Math. Monthly, 72(5), 500-506. https://doi.org/10.1080/00029890.1965.11970558

\section{Copyrights}

Copyright for this article is retained by the author(s), with first publication rights granted to the journal.

This is an open-access article distributed under the terms and conditions of the Creative Commons Attribution license (http://creativecommons.org/licenses/by/4.0/). 